SCADide

Infer, Interpret \& Inspire Science
International Journal of Dentistry and Oral Science (IJDOS)

ISSN: $2377-8075$

\title{
Evaluate the Effectiveness Of Vital Pulp Therapy Using Bioceramic Endosequense Root Repair Mate- rial (ERRM) compared to Enriched Mixture Cement (CEM) in Symptomatic Mature Permanent Posterior teeth: Clinical and Radiographical Study

Ameer Al-Kazzaz ${ }^{1}$, Hassan Ashour², Sharif Barakat ${ }^{3}$, Muaaz Alkhouli ${ }^{*}$

${ }^{1} \mathrm{PhD}$ Candidate, Department of Endodontics, Faculty of Dentistry, Damascus University, Damascus, Syria.

${ }^{2}$ Assistant Professor, Department of Endodontics, Faculty of Dentistry, Damascus University, Damascus, Syria.

${ }^{3}$ Professor, Department of Oral Pathology, Faculty of Dentistry, Damascus University, Damascus, Syria.

${ }^{4} \mathrm{PhD}$ Candidate, Department of Pediatric Dentistry, Faculty of Dentistry, Damascus University, Damascus, Syria.

\section{Abstract}

Objective: The aim of this study was to evaluate the efficacy of vital pulp therapy using bioceramic Endosequense Root Repair Material (ERRM) compared to Enriched Mixture Cement (CEM) in Mature permanent posterior teeth suffering from symptoms of non-reversible acute pulpitis due to caries.

Methods: The sample consisted of 60 permanent human posterior teeth suffering from non-reversible pulpitis in 53 male and female patients aged between 18-40 years. An assessment of the dental condition was conducted clinically and radiologically before starting the treatment based on: the presence of a history of the disease (persistent spontaneous pain that does not go away with the disappearance of the cause), the results of the cold test, in addition to the radiological findings. Vital pulp therapy was performed (partial or complete pulp amputation)after haemostasis using either (ERRM) or (CEM). The final restoration was applied in the same session, then an $\mathrm{x}$-ray radiograph was taken immediately after the treatment procedure. Patient were re-called after 24 hours to ensure the disappearance of symptoms and then according to the following time intervals: (a week, 3 months, 6 months, a year) to evaluate the cases clinically and radiographically.

Results: This study showed that there were no statistically significant differences in the frequencies of clinically and radiographically assessment results between the CEM group and ERRM group, in each of the studied time periods (after one week, after one month, after three months, after six months, after one year)

Conclusion: Vital pulp Therapy using ERRM and CEM showed a high success rate after a one-year observation period in permanent teeth suffering from non-response pulpitis, and it can be a staged alternative to traditional endodontic treatments.

Keywords: Vital Pulp Therapy; Non-Reversible Pulpitis; ERRM, CEM.

\section{Introduction}

Dental caries is one of the most challenging infectious diseases worldwide. Untreated caries can cause irreversible pulpitis, which require root canal therapy. Permanent mature teeth with irreversible pulpitis or carious pulp exposure with/without clinical/radiological findings of apical periodontitis should be treated by root canal therapy.[1] However, root canal treatment has many shortcomings: 1 - After a root canal procedure is performed on the tooth, the tooth will no longer be vital so it will be brittle. A root canal treated tooth will be more prone to fractures. 2- The procedure is too long and complicated, things that are uncomfortable for both the practitioner and the patient. 3- Root canal therapy can be considered an expensive treatment option.

According to many authors, an attempt should be made to provide less costly treatment options that are applicable in the above circumstances. Vital pulp therapy (VPT) can be provided as a less expensive, simple and effective treatment option. [2]

The aims of VPT include maintenance of the vitality of the dental pulp and stimulation of the remaining pulp tissue for adequate

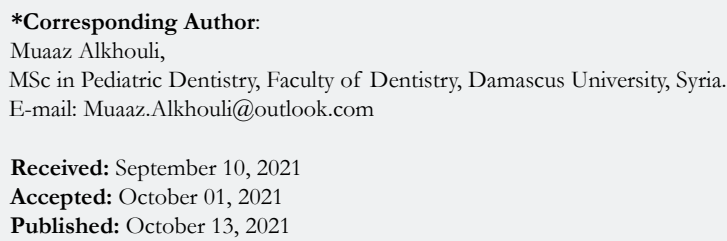

Citation: Ameer Al-Kazzaz, Hassan Ashour, Sharif Barakat, Muaaz Alkhouli. Evaluate the Effectiveness Of Vital Pulp Therapy Using Bioceramic Endosequense Root Repair Material (ERRM) compared to Enriched Mixture Cement (CEM) in Symptomatic Mature Permanent Posterior teeth: Clinical and Radiographical Study. Int J Dentistry Oral Sci. 2021;8(10):4758-4763. doi: http://dx.doi.org/10.19070/2377-8075-21000965

Copyright: Muaaz Alkhouli 2021 . This is an open-access article distributed under the terms of the Creative Commons Attribution License, which permits unrestricted use, distribution and reproduction in any medium, provided the original author and source are credited. 
structural and functional healing. The response of the pulp to such treatment can be summarized as follows; in the tissues adjacent to the exposure site, inflammatory cells, and extravascular erythrocytes can be observed. The initial damage results in the production of fibrinogen, formation of blood clot, and an acute inflammation accompanied by the presence of polymorphonuclear leukocytes (PMNs). Then, some particles from the pulpcovering material also enter the underlying pulp tissue, which cause hard tissue formation. [3]

Vital pulp therapy includes indirect pulp capping, direct pulp capping, partial pulpotomy and full pulpotomy. [4]

There are different capping materials that can be used while performing vital pulp therapy; calcium hydroxide, MTA, Resin Modified Glass Ionomers, Adhesive Resins, Bioceramics and Calcium Enriched Matrix. [5]

In this study, two biomaterials were studied and investigated in vital pulpotomy techniques. The materials studied were EndoSequence Root Repair Material (ERRM) and Calcium Enriched Matrix (CEM).[6]

EndoSequence Root Repair Material (ERRM) is one of the bioceramic materials that have been introduced recently as alternatives to MTA. Cytotoxicity of ERRM was similar to that for MTA according to many studies. Currently, there is limited research on the Endosequence Root Repair Material (ERRM). It has mainly been evaluated for use as a root-end filling material. Its properties include exceptional stability, high mechanical bond strength, high $\mathrm{pH}$, radiopaque, and hydrophilic setting properties, and it is a premixed material.[7]

Calcium enriched mixture (CEM) cement was introduced to dentistry as an endodontic filling biomaterial. The major components of the cement powder are calcium oxide $(\mathrm{CaO})$, sulfur trioxide (SO3), phosphorous pentoxide (P2O5), and silicon dioxide (SiO2). The physical properties of this biomaterial, such as flow, film thickness, and primary setting time are favorable, and its clinical applications are similar to those of MTA. [8]

CEM pulpotomy of symptomatic permanent teeth was also evaluated. In a case series study of 12 permanent mature molars with irreversible pulpitis, CEM was used for pulpotomy, and resulted in complete success at a 16-month follow-up. It was also shown that to enable improved regeneration, the pulp-dentin complex had isolated itself by forming a calcified bridge [9]. In a multicenter randomized clinical trial in 23 dental centers linked to five medical universities in Iran, pulpotomy treatment of mature permanent molars diagnosed with irreversible pulpitis was performed using CEM and MTA. The results of this trial showed high clinical/ radiographic success rates of CEM $(>92 \%)$ during a follow-up after one and two years.[10]

Conversely, to the best of our knowledge, there are no study that has been compared both ERRM and CEM in vital pulpotomy of permanent mature molars. For that reason, our study was aimed to compare between the two materials with a follow up period of 12 months.

\section{Material and Methods}

\section{Study design, setting and Sample}

The design of this trial was a clinical study with two interventional arms which was done to study the effectiveness of CEM compared to ERRM in vital pulpotomy in posterior permanent teeth with closed apex. The ethical approval was obtained from the ethical committee of Damascus university.

This study was conducted on a 60 posterior permanent teeth with closed apexes that suffer from carious acute pulpitis (carious exposure with a pathological history of spontaneous, persistent pain, and does not resolve with the disappearance of the causative factor). The sample consisted of 53 male and female patients, their ages ranged between (18-40) years, which was selected from patients visiting the Department of Endodontic at the Faculty of Dentistry - Damascus University. This study was done in the previously mentioned department.

The patient's written consent was taken to carry out the treatment and included it in the research, after informing him in detail about the research, its methods, its aim and the quality of the applied material, that the treatment used. In case of failure, the teeth will be endodontically treated in a traditional way.

Special forms were designed for this study, in which the diagnostic information of each patient was written down, in addition to general information about his health status and oral health, and included a table to record the studied cases, the materials used, and the developments that occurred in them according to the observation times.

The sample was randomly divided into two groups:

- The first group: (30) teeth, treated with vital pulpotomy using $\mathrm{CEM}$ in one session with the final restoration.

The second group: (30) teeth, treated with vital pulpotomy using ERRM in one session with the final restoration.

The sample as a whole was monitored at intervals of one week, one month, three months, six months, and 12 months.

\section{Inclusion and Exclusion Criteria}

1- The patient were in a healthy physical condition and didn't suffer from any history of illness or taking medications that are contraindicated for vital pulpotomy.

2- age group ranging between 18-40 years.

3- Patients who have exposed pulp as a result of trauma or fracture were excluded.

4- An apical x-ray radiograph was performed for all the eligible teeth on which the research was conducted before starting the treatment to know the general condition of the treated tooth, especially the condition of the periapical tissues and the junction area of the roots. The simple ligamentous expansion in the apical region was overlooked when the rest of the symptoms and signs indicated the diagnosis of the case as acute pulpitis, and the diagnosis was confirmed after opening the pulp chamber during vital pulpotomy.

5- To test pulp vitality, palpation and percussion test, ethyl chlorine (Cognoscin-AVEFLOR) test and the electric pulp tester were 
performed.

6- The integrity of the periodontal tissues was confirmed by probing them with the World Health Organization (WHO) probe, and the teeth that developed fistula in the oral cavity were excluded.

7- Teeth that were suffering from periodontal inflammation, or excessive movement of the tooth, and teeth with cracks visible to the naked eye or with clinically or radiologically obvious fractures were excluded.

8- Teeth that show symptoms of pulp necrosis, or that show internal or external absorption were excluded, and teeth in which bleeding from the exposed pulp did not stop after ten minutes of accidental exposure were also excluded. (Song, Kang et al. 2015).

\section{Study protocol}

An apical radiograph were taken for each case to know the general condition of the treated tooth, especially the condition of the periapical tissues. Patients were asked to use a mouthwash of chlorhexidine $2 \%$ for 5 minutes, then the eligible tooth was anesthetized by infiltration (for the teeth of the upper jaw) or with regional anesthesia (for the teeth of the lower jaw).

The tooth was isolated using a rubber dam, then the initial preparation was done using a high-speed handpiece, a sterile diamond bur with abundant air-water cooling, then the caries were excavated by a slow rotary handpiece to remove the caries on the walls of the cavity without approaching the pulpal wall.

After pulpal exposure, a sterile bur was used to perform a partial pulp amputation and remove the inflamed pulpal tissue with no more than $2-3 \mathrm{~mm}$ of the coronary pulp in the place of exposure, then chlorhexidine was used to wash the cavity to reduce bacterial virulence, taking into account the following:

1. The exposed pulp shows symptoms of recent and fresh bleeding.

2. Control of bleeding within a period of 8-10 minutes which was carried out by continuous washing with saline solution and then applying a moist cotton ball to the pulp wound site without pressure. If the bleeding was not controlled, the tooth was excluded from the study. If the exposed coronary pulp tissue didn't not show any signs of bleeding, the coronary pulp tissue was considered necrotic and the tooth was also excluded from the sample.
After that, the cavity was washed with 17\% EDTA solution for 1 minute to remove any residual excavated dentin from the preparation (to prevent the introduction of dentinal crumbs into the pulpal wound area) and to condition the dentinal surface, which will improve the adhesion of the biomaterial used in the coating in addition to its gram-positive and gram-negative antibacterial action and its role in releasing growth factors from the dentinal canal.

The cavity was re-washed with saline, then the amputated pulp was covered with one of the two materials studied in the research (CEM, ERRM) according to the instructions of the manufacturer for each material, so that a homogeneous coverage was obtained of the pulp wound with a thickness of $2 \mathrm{~mm}$ from the biomaterial and without applying pressure.

After Resin modified glass ionomer cement has been applied. Then, a final restoration was placed in the same session, then a radiograph was taken immediately after the treatment.

\section{Follow-up and Outcome measures}

The patient was recalled after 24 hours to ensure the relieving of symptoms and then thery were also recalled according to the following time intervals: (a week, a month, 3 months, 6 months, a year), and the completed cases were evaluated in the periodic review sessions by:

1- Questioning the patients and inquiring about the symptoms they felt after treatment.

2- Checking the quality of the coronary restoration, which was repaired, if necessary.

3- Conducting clinical and radiological examinations to assess the condition of the pulp and periapical tissues, including:

A - Examination of the soft tissues for the presence of a bulge, a fistula or a gingival pocket.

b- Examination of the treated tooth to investigate the movement of the tooth or the occurrence of pain upon palpation or percussion to investigate the presence of inflammation in the periapical tissues.

C- Dental pulp vitality tests were done by external electrical stimuli using an electric pulp tester or cold using chloroethyl. The presence of a response upon examination indicates the presence of vitality in the pulp tissue in the coronary region, but it does

Figure 1. Shows the tooth after preparation and removal of the missing part.

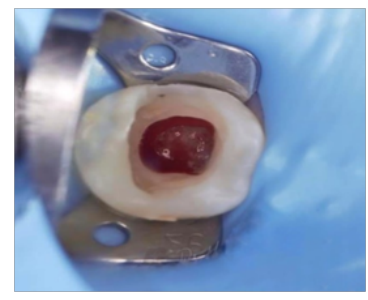

Figure 2. Shows the pulp tissue after stopping the bleeding.

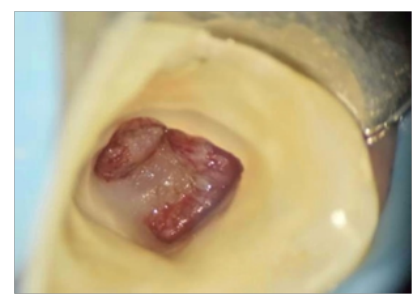


not indicate the degree of inflammation, and the absence of a response indicates the presence of necrosis (local or complete).

D- Radiographic examination: X-ray radiographs were performed using the oral sensor according to the observation periods (3 months, 6 months, 12 months)

- Two blinded specialists in endodontic were also asked to assess the status of the periapical tissues on the $\mathrm{x}$-ray radiographs, that were taken at intervals of 3 months, 6 months, 12 months, using the Periapical Index Scoring System (PAI) developed by Ørstavik [10] as following:

Criteria for success and failure in the clinical radiographical study Vital pulp therapywas considered successful if the following criteria were achieved:

1. Pulp was showing positive response in the vital tests with the absence of symptoms and clinical signs of pulpal inflammation or necrosis (pain, pain on percussion, presence of fistula, swelling).

2. There were no signs of pathological injury on the radiograph (root resorption, dislocation or new questioned appearance in the apex or junction of the roots)

3. Complete periapical radiographical healing with a score of 1 or 2 on the PAI scale, or a decrease in radiographic transparency if present before the treatment.

Whereas, the vital pulp therapy was consideredas a failure, if clinical or radiographic symptoms or signs appeared, such as sensitivity to biting, the presence of a fistula or soft tissue swelling related to the treated tooth, the development of symptoms of acute pulpitis, or development of a periapical lesion or in the area of root junction or internal absorption, in addition to signs of vertical or horizontal root fractures.

\section{Results}

A total of 60 permanent molars were enrolled in this study. Each molar was assessed by the examiner both clinically and radiographically in five different time intervals.

The PAI radiological assessment score and clinical status score were monitored and the treatment outcome was determined clinically and radiographicallyin terms of success and failure at five different time intervals (after one week, after one month, after three months, after six months, after one year) for each case of Cases of treatment in the research sample.

\section{Results of the radiographical assessment}

Periapical Index scoring system was used to evaluate the radiological success rate of the treated molars. Teeth that has the score of PAI $>2$ were considered failed radiologically.

It is noted that during the time periods studied in the research, all cases were successful radiologically regardless of the material used. Therefore, this study showed that there are no statistically significant differences in the frequencies of radiological assessment result between the CEM group and ERRM group, in each of the studied time periods (after one week, after one month, after three months, after six months, after one year) separately in the research sample.

In addition, it was concluded that there are no statistically significant differences in the frequencies of radiological treatment

Table 1. Periapical Index Scoring System (PAI).

\begin{tabular}{|c|c|}
\hline Score & Criteria \\
\hline 1 & Normal periapical structures \\
\hline 2 & Small changes in bone structures \\
\hline 3 & Changes in bone structure with some mineral loss \\
\hline 4 & Periodontitis with well-defined radiolucent area \\
\hline 5 & Severe periodontitis with exacerbating features \\
\hline
\end{tabular}

Teeth with a PAI score of $>2$ were considered as failure.

Table 2. Frequencies of clinical success and failure cases in the research sample.

\begin{tabular}{|c|c|c|c|c|}
\hline $\begin{array}{c}\text { Follow-up } \\
\text { appointment }\end{array}$ & Study Group & $\begin{array}{c}\text { Number of Clini- } \\
\text { cal success cases }\end{array}$ & $\begin{array}{c}\text { Number of Clin- } \\
\text { ical failure cases }\end{array}$ & sum \\
\hline \multirow{2}{*}{ After 1 week } & CEM & 0 & 30 & 30 \\
\cline { 2 - 5 } & ERRM & 0 & 30 & 30 \\
\hline \multirow{2}{*}{ After 1 month } & CEM & 0 & 30 & 30 \\
\cline { 2 - 5 } & ERRM & 1 & 29 & 30 \\
\hline \multirow{2}{*}{ After 3 months } & CEM & 1 & 29 & 30 \\
\cline { 2 - 5 } & ERRM & 1 & 28 & 29 \\
\hline \multirow{2}{*}{ After 6 months } & CEM & 0 & 29 & 29 \\
\cline { 2 - 5 } & ERRM & 0 & 28 & 28 \\
\hline \multirow{2}{*}{ After 1 year } & CEM & 0 & 29 & 29 \\
\cline { 2 - 5 } & ERRM & 0 & 28 & 28 \\
\hline
\end{tabular}


result between the five studied periods (after one week, after one month, after three months, after six months, after one year) in both the CEM group and the ERRM material group separately in the research sample.

\section{Results of the clinical assessment}

Chi-square test was conducted to study the significance of the differences in the frequencies of treatment result clinically between the CEM group and the ERRM group in the research sample.

It was shown that the value of the significance level is greater than 0.05 after one month and after three months. Therefore, at the $95 \%$ confidence level, there are no statistically significant differences in the frequencies of the treatment result clinically after one month and after three months between the CEM group and the ERRM group in the research sample.

McNemar test was conducted to study the significance of binary differences in the frequency of treatment result clinically among the five studied periods (after one week, after one month, after three months, after six months, after one year) in the research sample.

McNemar test showed that there are no statistically significant bilateral differences in the frequencies of treatment result clinically between the respective periods in the research sample, noting that all failures in both subjects were in the first three months of treatment.

\section{Discussion}

Vital pulpotomy is defined as the surgical removal of thecoronal pulp tissue to preserve the remaining root pulp tissue. [11] The inflamed pulp tissue is removed until a healthy pulp tissue level is reached. [12]

This study was aimed to compare between two types of the biomaterials; CEM and ERRM while performing vital pulpotomy in mature permanent teeth. In the present study, we evaluated the success rate of both materials clinically and radiographically.

This study was carried out on patients whose ages ranged between 18-40 years, and therefore they are from one age group that does not belong to young or old ages, in which the vascularization of the dental pulp, the diameter of the apex, or the transformational changes can affect the ability of the underlying pulp to heal positively or negatively. [13]

The good prognosis in vital pulp therapy depends on the removal of all pathological factors. For that reason, it is preferable when performing such treatment when the endodontic covering and the final restoration can be applied directly to reduce the risk of subsequent infection and damage of the dental pulp.[14]

Therefore, our study relied on the completion of clinical procedure in both groups in one session in order to avoid the occurrence of microleakage that could affect the results of the study. Kim and his colleagues mentioned in 2015 that the covering material used in endodontic coverage does not cover the entire dentinal canals exposed as a result of preparing the cavity. The dental cavity must be sealed with a material that prevents marginal leakage, which is a path for germs towards the dental pulp. [16]

The study also mentioned that temporary fillings of all kinds are not able to prevent marginal leakage completely, and the amount of leakage decreased when the thickness of the temporary filling was about $3 \mathrm{~mm}$, but there is still a passage that allows germs to pass through the dental pulp. Therefore, the current study was keen to avoid this problem by carrying out coverage procedures in one session.[17]

With increasing evidence about the possibility of preserving the vitality of the pulp tissue, contrary to what was previously believed, in addition to the great development in the field of bioceramic materials and their wide uses in the field of dental treatment. This experimental study was carried out using the vitalpulptherapy (vital pulpotomy) on mature permanent teeth (showing symptoms of irreversible pulpitis) $[18,19]$.

As a result of the present study, There was no effect of the material used on the success of the treatment, so vital pulp therapy can be considered as a successful treatment procedure in the mature permanent teeth that show symptoms of irreversible pulpitis.

The clinical and radiographical assessment showed high success rates, reaching $(96.6 \%)$ for CEM and $(93.3 \%)$ for ERRM after one year of treatment.

In addition, no cases that reported clinical failure did show radiographical changes (they were successful radiographically).

The success rate of vital pulpotomy in this study for each of the two studied materials was close with no statistically significant difference. This can be because they are biologically active materials from hydraulic calcium silicate based cements, and both materials have similar biological properties when used in vital pulp therapy. In addition,the high biocompatibility of the materials used, their stimulating effect on the cells of the endoblast, the generation of new blood vessels, their bactericidal properties and theirstimulation of the formation of a dentinal bridge. [20-22]

The results of our study was in accordance with Asgary's study that has been conducted in 2013 on mature permanent carious teeth with symptoms of irreversible pulpitis. Vital pulpotomy was performed using MTA and CEM materials with a sample size of 413 teeth, for patients aged between 9-65 years. The treatment success rate was $92 \%$ for CEM and $95 \%$ for MTA after one year of follow-up. [23, 24]

Another study published by Asgary in 2015 to evaluate the success of vital pulp therapy in mature permanent teeth using CEM with a follow-up period that extended to five years. The study included 407 teeth. The sample was randomly divided into two groups, the first group included 205 teeth, a vital pulpotomy was performed using CEM, the second group included 202 teeth and rootcanal treatment was performed. The success of vital pulp therapy using CEM was $78.1 \%$, while the success rate of conventional endodontic treatment was 75.3\%.Therefore, Asgary suggested that vital pulp therapy using CEM can be an acceptable alternative to rootcanal treatment in mature permanent teeth with symptoms of irreversible pulpitis.[25] 
In addition, our study agreed with the results of the systematic review conducted by Firas Elmsmari and his colleagues in 2019, in which they stated that vital pulpotomy is an appropriate treatment option when treating posterior permanent teeth that have been exposed to carious exposures and suffer from pulpitis (reversible or irreversible).[26]

\section{Conclusion}

Vital pulp Therapy using ERRM and CEM showed a high success rate after a one-year observation period in permanent teeth suffering from non-response pulpitis, and it can be a staged alternative to traditional endodontic treatments.

\section{Acknowledgment}

Damascus university funded this research.

\section{References}

[1]. Chin JS, Thomas MB, Locke M, Dummer PM. A survey of dental practitioners in Wales to evaluate the management of deep carious lesions with vital pulp therapy in permanent teeth. Br Dent J. 2016 Sep 23; 221(6): 3318. Pubmed PMID: 27659637.

[2]. Schwendicke F, Stolpe M. Direct pulp capping after a carious exposure versus root canal treatment: a cost-effectiveness analysis. J Endod. 2014 Nov; 40(11): 1764-70. PMID: 25218524.

[3]. Cvek M. A clinical report on partial pulpotomy and capping with calcium hydroxide in permanent incisors with complicated crown fracture. J Endod. 1978 Aug; 4(8): 232-7. PMID: 283188

[4]. Levin LG, Law AS, Holland GR, Abbott PV, Roda RS. Identify and define all diagnostic terms for pulpal health and disease states. J Endod. 2009 Dec; 35(12): 1645-57. PMID: 19932339.

[5]. Matsuo T, Nakanishi T, Shimizu H, Ebisu S. A clinical study of direct pulp capping applied to carious-exposed pulps. J Endod. 1996 Oct; 22(10): 5516. PMID: 9198445.

[6]. Aguilar P, Linsuwanont P. Vital pulp therapy in vital permanent teeth with cariously exposed pulp: a systematic review. J Endod. 2011 May; 37(5): 5817. PMID: 21496652

[7]. Asgary S, Eghbal MJ, Fazlyab M, Baghban AA, Ghoddusi J. Five-year results of vital pulp therapy in permanent molars with irreversible pulpitis: a noninferiority multicenter randomized clinical trial. Clin Oral Investig. 2015 Mar; 19(2): 335-41. PMID: 24771228.

[8]. Taha NA, Ahmad MB, Ghanim A. Assessment of Mineral Trioxide Aggregate pulpotomy in mature permanent teeth with carious exposures. Int Endod J. 2017 Feb; 50(2): 117-125. PMID: 26715408.

[9]. Glickman GN. AAE Consensus Conference on Diagnostic Terminology: background and perspectives. J Endod. 2009 Dec; 35(12): 1619-20. PMID: 19932336

[10]. SELTZER S, BENDER IB, ZIONTZ M. THE DYNAMICS OF PULP
INFLAMMATION: CORRELATIONS BETWEEN DIAGNOSTIC DATA AND ACTUAL HISTOLOGIC FINDINGS IN THE PULP. Oral Surg Oral Med Oral Pathol. 1963 Aug; 16: 969-77. PMID: 14049126.

[11]. Dummer PM, Hicks R, Huws D. Clinical signs and symptoms in pulp disease. Int Endod J. 1980 Jan; 13(1): 27-35. PMID: 6935168.

[12]. Bjørndal L. The caries process and its effect on the pulp: the science is changing and so is our understanding. Pediatr Dent. 2008 May-Jun; 30(3): 192-6. PMID: 18615983

[13]. Ricucci D, Loghin S, Siqueira JF. Correlation between clinical and histologic pulp diagnoses. J Endod. 2014 Dec; 40(12): 1932-9. PMID: 25312886.

[14]. Guideline on Pulp Therapy for Primary and Immature Permanent Teeth. Pediatr Dent. 2016 Oct; 38(6): 280-288. PMID: 27931467.

[15]. Witherspoon DE. Vital pulp therapy with new materials: new directions and treatment perspectives--permanent teeth. J Endod. 2008 Jul; 34 (7 Suppl): S25-8. PMID: 18565368.

[16]. Nair PN, Duncan HF, Pitt Ford TR, Luder HU. Histological, ultrastructural and quantitative investigations on the response of healthy human pulps to experimental capping with mineral trioxide aggregate: a randomized controlled trial. Int Endod J. 2008 Feb; 41(2): 128-50. PMID: 17956562.

[17]. Min KS, Park HJ, Lee SK, Park SH, Hong CU, Kim HW, et al. Effect of mineral trioxide aggregate on dentin bridge formation and expression of dentin sialoprotein and heme oxygenase-1 in human dental pulp. J Endod. 2008 Jun; 34(6): 666-70. PMID: 18498885.

[18]. Asgary S, Eghbal MJ, Parirokh M, Ghanavati F, Rahimi H. A comparative study of histologic response to different pulp capping materials and a novel endodontic cement. Oral Surg Oral Med Oral Pathol Oral Radiol Endod. 2008 Oct; 106(4): 609-14. PMID: 18718783.

[19]. aller KM, Buchalla W, Hiller KA, Federlin M, Eidt A, Schiefersteiner M, et al. Influence of root canal disinfectants on growth factor release from dentin. J Endod. 2015 Mar; 41(3): 363-8. PMID: 25595468.

[20]. Qrstavik D, Radiology of apical periodontitis. IN: Qrstavik D,Pitt ford Tr . Essential endodontology .prevention and treatment of apical periodontitis, 1st edn xford: Blackwell Scince ltd, 1998.

[21]. Song M, Kang M, Kim HC, Kim E. A randomized controlled study of the use of ProRoot mineral trioxide aggregate and Endocem as direct pulp capping materials. J Endod. 2015 Jan; 41(1): 11-5. PMID: 25443279.

[22]. Chailertvanitkul P, Paphangkorakit J, Sooksantisakoonchai N, Pumas N, Pairojamornyoot W, Leela-Apiradee N, et al. Randomized control trial comparing calcium hydroxide and mineral trioxide aggregate for partial pulpotomies in cariously exposed pulps of permanent molars. Int Endod J. 2014 Sep; 47(9): 835-42. PMID: 24299006.

[23]. Taha NA, Khazali MA. Partial Pulpotomy in Mature Permanent Teeth with Clinical Signs Indicative of Irreversible Pulpitis: A Randomized Clinical Trial. J Endod. 2017 Sep; 43(9): 1417-1421. PMID: 28673494.

[24]. Simon S, Smith AJ, Lumley PJ, Cooper PR, Berdal A. The pulp healing process: from generation to regeneration. Endodontic Topics. 2012 Mar; 26(1): 41-56.

[25]. Simon S, Perard M, Zanini M, Smith AJ, Charpentier E, Djole SX, et al. Should pulp chamber pulpotomy be seen as a permanent treatment? Some preliminary thoughts. Int Endod J. 2013 Jan; 46(1): 79-87. PMID: 22900881.

[26]. Mente J, Hufnagel S, Leo M, Michel A, Gehrig H, Panagidis D, et al. Treatment outcome of mineral trioxide aggregate or calcium hydroxide direct pulp capping: long-term results. J Endod. 2014 Nov; 40(11): 1746-51. PMID: 25227216. 